\title{
Pimarane Diterpenoids from Aerial Parts of Lycopus lucidus and Their Antimicrobial Activity
}

\author{
Jitendra Pandey $\mathbb{D}^{1},{ }^{1}$ Bang Yeon Hwang, ${ }^{2}$ Hyeong-Kyu Lee, ${ }^{3}$ and Amrit Poudel $\mathbb{D}^{4}$ \\ ${ }^{1}$ Department of Pharmacy, Crimson College of Technology, Pokhara University, Devinagar-11, Butwal 32900, Nepal \\ ${ }^{2}$ Chungbuk National University, Department of Pharmacy, Cheongju, Republic of Korea \\ ${ }^{3}$ Natural Medicine Research Center, Korea Research Institute of Bioscience \& Biotechnology, \\ Cheongju-si 363-883, Republic of Korea \\ ${ }^{4}$ Department of Biodiversity and Bioresources, Satvik Nepal, Dandakonak, Kaski, Pokhara 33700, Nepal
}

Correspondence should be addressed to Amrit Poudel; amritpoudel@gmail.com

Received 17 October 2021; Accepted 18 January 2022; Published 4 February 2022

Academic Editor: Rafael M. Ximenes

Copyright (c) 2022 Jitendra Pandey et al. This is an open access article distributed under the Creative Commons Attribution License, which permits unrestricted use, distribution, and reproduction in any medium, provided the original work is properly cited.

\begin{abstract}
The ethyl acetate fraction obtained from aerial parts of $L$. lucidus was subjected for isolation of new bioactive compounds, which enabled isolation of five new pimarane-type diterpenoids, namely, $3 \beta, 8 \beta, 12 \beta, 18$-tetrahydroxy pimar-15-ene $(\mathbf{1 0}), 7 \alpha, 8 \beta, 12 \beta, 18$ tetrahydroxy pimar-15-ene (11), 3 $\beta, 8 \beta, 11 \beta, 12 \alpha, 18$-pentahydroxy pimar-15-ene (12), $12 \beta$ acetoxy, $8 \beta, 3 \beta, 18$-trihydroxy pimar15 -ene (13), and $3 \beta$ acetoxy, $8 \beta, 12 \beta, 18$-trihydroxy pimar-15-ene (14), along with nine known compounds. The structures were elucidated by spectroscopic analysis and comparison with literature data. The isolated new pimarane diterpenoids were examined for antimicrobial activity against Gram-negative and Gram-positive bacteria strains. Among them, the compound $3 \beta, 8 \beta, 12 \beta, 18$ tetrahydroxy pimar-15-ene (10) was most effective, exhibiting minimum inhibitory concentration (MIC) values of $15.62 \mu \mathrm{g} / \mathrm{mL}$ against Staphylococcus epidermidis, $31.25 \mu \mathrm{g} / \mathrm{mL}$ against Staphylococcus aureus, $62.5 \mu \mathrm{g} / \mathrm{mL}$ against Pseudomonas aeruginosa, and $125 \mu \mathrm{g} / \mathrm{mL}$ against Escherichia coli.
\end{abstract}

\section{Introduction}

The genus Lycopus of family Lamiaceae (Labiatae) contains around 16 species with wide distribution in Europe, Asia, and North America [1]. In Asia, Lycopus lucidus is widely distributed species in Korea, China, Japan, Russia, and Taiwan. It is most abundant in Korea. L. lucidus is a flowering perennial glabrous herb occurring in aquatic environment and grows up to $0.6-1.2 \mathrm{~m}$ height at an altitude of $320 \mathrm{~m}$ to $2100 \mathrm{~m} \mathrm{[2-4].} \mathrm{L.} \mathrm{lucidus} \mathrm{is} \mathrm{one} \mathrm{of} \mathrm{the} \mathrm{popular}$ edible plants with its long history as a folk remedy in traditional medicinal system of China, Japan, and Korea [5]. This plant has been used as both traditional and official formulations, as they are potent source of bioactive tannins, coumarins, flavonoids, terpenoids, and essential oils. Major bioactive compounds that have been isolated from this plant are flavonoid and its esters, rosmarinic acid derivatives, phenylpropanoids, steroids, pentacyclic triterpenes, essential oils, oligosaccharides, polysaccharides, and diterpenoid glycosides [3, 6-8]. The leaf and stem (aerial part) of L. lucidus have been extensively used for the treatment of inflammation, cardiovascular problem, insomnia, menstrual problems, and thyroid problem, as a sedative, wound healing, pain reliving agents, herbal tea, and useful tonic $[6,8,9]$. The root of $L$. lucidus is known as small ginseng in China and widely used as dietary supplement [10]. Many biological activities such as inhibition of superoxide radical [4], nitric oxide scavenging effect [6], inhibition of hypercholesterolemia and atherosclerosis [11], acaricidal activities [10], and hyaluronidase inhibition [5] have been explored from this plant.

In the current scenario, bacterial infectious diseases are a serious worldwide public health problem due to an increase in their resistance towards antibiotics, which have ultimately 
given on to the birth of multiresistant bacterial strains. Increased rates of mortality and morbidity are due to the lack of long-term effective drugs and the unaffordable cost of new generation antibiotics. The problem of microbial resistance is growing and the prospect of the use of antimicrobial drugs is uncertain. This disastrous situation has compelled us to explore more successful antimicrobial agents using plant resources so that they will serve as an active therapeutic ingredient, as well as leading molecule for the synthesis of optimized new drugs. The plant species have always been serving as a major source of novel and potent antimicrobial constituents, as they possess the capability to synthesize secondary metabolites to combat diverse pathogenic microorganisms available in the environment [12]. In this context, our study is mainly focused on isolation and structure elucidation of possible new compounds from the aerial parts of $L$. lucidus followed by screening their antibacterial properties against pathogenic Gram-positive and Gram-negative bacterial strains.

\section{Materials and Methods}

2.1. General Experimental Procedure. Analysis of NMR spectra was carried out through Varian UNITY 400 (Varian, Inc., Palo Alto, CA) FT-NMR spectrometer using the tetramethylsilane as an internal standard. Waters Q-Tof Premier spectrometer (Micromass UK Ltd., Manchester, UK) was used to obtain HR-ESI mass spectra. Sephadex LH-20 (25-100 $\mu \mathrm{m}$, Sigma-Aldrich, Steinheim, Germany), silica gel (230-400 mesh, SiliCycle Inc., Quebec, Canada), and RPC18 (Cosmosil 40 $\mathrm{C}_{18}$-PREP, Kyoto, Japan) were used for column chromatography. Thin layer chromatography (TLC) was performed on precoated Kiesel-gel $60 \mathrm{~F}_{254}(0.25 \mathrm{~mm}$, Merck, Darmstadt, Germany) and Kiesel-gel 60 RP- $18 \mathrm{~F}_{254 \mathrm{~s}}$ (0.25 mm, Merck, Steinheim, Germany). HPLC analysis was conducted on Thermo Dionex Ultimate 3000 equipped with Ultimate 3000 pump, autosampler, column compartment and diode array detector and Agilent Technology model 1260 Infinity equipped with 1260 DAD. Columns used for the analysis were YMC Trait C18 ExRS $(5 \mu \mathrm{m}$, $4.6 \times 250 \mathrm{~mm})$, Phenomenex synergy RP-Polar $(5 \mu \mathrm{m}$, $4.6 \times 250 \mathrm{~mm})$, Thermo acclaim polar advantage $(5 \mu \mathrm{m}$, $4.6 \times 250 \mathrm{~mm})$, and Atlantis T3 $(5 \mu \mathrm{m}, 4.6 \times 250 \mathrm{~mm})$. Preparative HPLC was carried out with a model Gilson PLC 2020. Preparative columns were Thermo acclaim polar advantage $(5 \mu \mathrm{m}, 21.2 \times 250 \mathrm{~mm})$, Atlantis $(5 \mu \mathrm{m}$, $19 \times 250 \mathrm{~mm})$, and YMC Trait C18 ExRS $(5 \mu \mathrm{m}$, $20 \times 250 \mathrm{~mm}$ ).

2.2. Plant Material. The aerial parts of $L$. lucidus were collected from 'a private herb garden' in Yuseong-gu, Daejeon, Republic of Korea (geographical coordinates: $36.3622^{\circ} \mathrm{N}, 127.3561^{\circ} \mathrm{E}$ ), in May 2016. The taxonomical identification of the plant was confirmed by Prof. Ki Hwan Bae, Chungnam National University. A voucher specimen (KRIB0071766) was deposited at the herbarium of the Korea Research Institute of Bioscience and Biotechnology (KRIBB).
2.3. Extraction and Isolation from Aerial Parts of L. lucidus. Air-dried aerial parts of L. lucidus ( $3.5 \mathrm{~kg}$ ) were extracted five times by cold maceration with methanol $(9 \mathrm{~L})$ at room temperature, and $388 \mathrm{~g}$ of a solid extract was obtained. The methanol extract $(388 \mathrm{~g}$ ) was suspended in distilled water and then partitioned with hexane, ethyl acetate, and watersaturated butanol, which yielded $60 \mathrm{~g}$ of hexane-soluble extract, $49 \mathrm{~g}$ of ethyl acetate soluble extract, $45 \mathrm{~g}$ of butanol soluble extract, and $142 \mathrm{~g}$ of an aqueous extract. From the data of HPLC and TLC, the ethyl acetate fraction was shown to contain major compounds such as terpenoids and phenolics. The ethyl acetate fraction $(35 \mathrm{~g})$ was applied to reverse phase open column $(10.5 \mathrm{~cm} \times 35 \mathrm{~cm}, 2.8 \mathrm{~kg}$ of RP-C18 gel $)$ and eluted with a stepwise gradient of methanol in distilled water $(20 \%, 30 \%, 40 \%, 50 \%, 60 \%, 70 \% 80 \%$, and $90 \%$ methanol, each $8 \mathrm{~L}$ ), yielding 10 subfractions on the basis of TLC pattern (denoted by SR-EA-F1 to SR-EA-F10. Compound (1) $(29 \mathrm{mg})$ was obtained from fraction SR-EA-F3 (455 mg) using Preparative Liquid Chromatography PLC (5\%-50\% acetonitrile, gradient, Thermo acclaim polar advantage $5 \mu \mathrm{m}, 250 \times 21.2 \mathrm{~mm})$. Compound (2) $(13 \mathrm{mg})$ and compound (3) (11 mg) were separated from fraction SR-EAF4 $(579 \mathrm{mg}$ ) through PLC (5\%-95 acetonitrile, gradient, Thermo acclaim polar advantage $5 \mu \mathrm{M}, 250 \times 21.2 \mathrm{~mm}$ ). The compound (4) (17 mg) was isolated from the fraction SREA-F5 (345 mg) using PLC again (7\%-75 acetonitrile, gradient, Thermo acclaim polar advantage $5 \mu \mathrm{M}$, $250 \times 21.2 \mathrm{~mm})$. Fraction SR-EA-F6 (412 mg) was subjected to Sephadex LH-20 (100\% methanol as an eluent) to yield compound (5) $(41 \mathrm{mg})$. PLC analysis (7\%-75\% acetonitrile gradient, YMC Trait C18 ExRS $5 \mu \mathrm{M}, 250 \times 20 \mathrm{~mm}$.) of fraction SR-EA-F7 $(932 \mathrm{mg})$ resulted in impure fraction SREA-F7.1 (575 mg) and pure compound (8) $(15 \mathrm{mg})$. Fraction SR-EA-F7.1 again was subjected to PLC $(5 \%-70 \%$ acetonitrile gradient, YMC Trait C18 ExRS $5 \mu \mathrm{M}, 250 \times 20 \mathrm{~mm}$ ) to give compound (6) $(93 \mathrm{mg}$ ) and compound (7) $(115 \mathrm{mg})$. Fraction SR-EA-F8 (899 mg) was subjected to Sephadex LH$20(100 \%$ methanol as an eluent) to give UV active subfraction SR-EA-F8.1 (456 mg) and UV-nonactive subfraction SR-EA-F8.2 (220 mg). Compound (9) $(123 \mathrm{mg})$ was obtained from subfraction SR-EA-F8.1 by PLC analysis (5\%-95\% acetonitrile gradient, YMC Trait C18 ExRS $5 \mu \mathrm{M}$, $250 \times 20 \mathrm{~mm}$ ). From subfraction SR-EA-F8.2, compound (10) $(125 \mathrm{mg})$ and compound (11) (2.5 mg) were obtained through normal phase open column chromatography $(5 \mathrm{~cm} \times 45 \mathrm{~cm} ; 300 \mathrm{~g}$ of silica gel; hexane: EtOAc at $3: 7)$ and fraction SR-EA-F9 $(279 \mathrm{mg})$ and fraction SR-EA-F10 $(310 \mathrm{mg})$ were also subjected to normal phase open column chromatography $(5 \mathrm{~cm} \times 45 \mathrm{~cm} ; 300 \mathrm{~g}$ of silica gel; hexane: EtOAc at $4: 6$ and $5 \mathrm{~cm} \times 45 \mathrm{~cm} ; 300 \mathrm{~g}$ of silica gel; hexane: EtOAc at $1: 1$, respectively). Fraction SR-EA-F9 resulted in compound (12) (21 mg) whereas compound (13) (25 mg) and compound (14) (3 mg) were isolated from fraction SREA-F10 using same method used for compound (13).

2.3.1. Protocatechuic Acid (1). White gray crystalline powder; HR-ESI-MS $m / z: 153.12[\mathrm{M}-\mathrm{H}]-.{ }^{1} \mathrm{H}-\mathrm{NMR}(400 \mathrm{MHz}$, DMSO- $\left.d_{6}\right) \delta: 7.33(1 \mathrm{H}, d, J=2.0 \mathrm{~Hz}, \mathrm{H}-2), 7.28(1 \mathrm{H}, \mathrm{dd}$, 
$J=2.0,8.2 \mathrm{~Hz}, \mathrm{H}-6), 6.77(\mathrm{H}, d, J=8.0 \mathrm{~Hz}, \mathrm{H}-5) .{ }^{13} \mathrm{C}-\mathrm{NMR}$ $\left(100 \mathrm{MHz}, \mathrm{DMSO}-d_{6}\right) \delta: 167.3(-\mathrm{COOH}, \mathrm{C}-7), 155.0(\mathrm{C}-4)$, 144.9 (C-3), 121.9 (C-5), 121.7 (C-1), 116.5 (C2), 115.1 (6).

2.3.2. Protocatechualdehyde (2). White gray crystalline powder; HR-ESI-MS $m / z$ : 137.0251[M-H]-. ${ }^{1} \mathrm{H}-\mathrm{NMR}$ $\left(400 \mathrm{MHz}, \mathrm{DMSO}-d_{6}\right) \delta: 9.69(1 \mathrm{H}, \mathrm{s}, \mathrm{H}-7), 7.25(1 \mathrm{H}, d$, $J=8.0 \mathrm{~Hz}, \mathrm{H}-6), 7.22$ (1H, s, H-2), 6.90 (H, s, H-5). ${ }^{13} \mathrm{C}-\mathrm{NMR}$ $\left(100 \mathrm{MHz}, \mathrm{DMSO}-d_{6}\right) \delta: 190.0$ (-CHO, C-7), 152.1 (C-4), 145.8 (C-3), 129.8 (C-5), 124.49 (C-1), 115.5 (C2), 114.3 (6).

2.3.3. Methyl 3,4 Dihydroxy Benzoate (3). White gray crystalline powder; HR-ESI-MS $m / z: 167.148[\mathrm{M}-\mathrm{H}]-.{ }^{1} \mathrm{H}-$ NMR $\left(400 \mathrm{MHz}, \mathrm{DMSO}-d_{6}\right) \delta: 7.43(1 \mathrm{H}, \mathrm{dd}, J=2.0,8.0 \mathrm{z}$, H-6), 7.42 (1H, s, H-2), $6.83(1 \mathrm{H}, d, J=8.8 \mathrm{~Hz}, \mathrm{H}-5), 3.801$ $\left(3 \mathrm{H}, \mathrm{s}-\mathrm{OCH}_{3}, \mathrm{H}-8\right) .{ }^{13} \mathrm{C}-\mathrm{NMR}(100 \mathrm{MHz}, \mathrm{DMSO}-d 6) \delta$ : 167.12 (Carbonyl carbon, C-7), 151.01 (C-4), 147.18 (C-3), 123.44 (C-1), 121.74 (C-6), 114.98 (C-5), 112.71 (C-2), $55.526\left(-\mathrm{OCH}_{3}, \mathrm{C}-8\right)$.

2.3.4. Para-Hydroxy Benzoic Acid (4). White crystalline powder; HR-ESI-MS $m / z$ : $137.12[\mathrm{M}-\mathrm{H}]-. \quad{ }^{1} \mathrm{H}-\mathrm{NMR}$ $\left(400 \mathrm{MHz}, \mathrm{DMSO}-d_{6}\right) \delta: 7.78(2 \mathrm{H}, d, J=8.5 \mathrm{~Hz}, \mathrm{H}-3 \& \mathrm{H}-5)$, $6.81(2 \mathrm{H}, d, J=8.4 \mathrm{~Hz}, \mathrm{H}-2 \& \mathrm{H}-6) .{ }^{13} \mathrm{C}-\mathrm{NMR}(100 \mathrm{MHz}$, DMSO-d6) $\delta: 167.1$ (-COOH), 161.5 (C-4), 131.5 (C-2\& C6), 121.4 (C-1), 115.0 (C-3\&C5).

2.3.5. Caffeic Acid (5). White crystalline powder; HR-ESIMS $m / z: 179.159[\mathrm{M}-\mathrm{H}]-.{ }^{1} \mathrm{H}-\mathrm{NMR}\left(400 \mathrm{MHz}\right.$, Methanol- $\left.d_{4}\right)$ $\delta: 7.55(1 \mathrm{H}, d, J=15.6 \mathrm{~Hz}, \mathrm{H}-7), 7.07(1 \mathrm{H}, d, J=2.0 \mathrm{~Hz}, \mathrm{H}-2)$, $6.95(1 \mathrm{H}, \mathrm{dd}, J=2.0 \mathrm{~Hz}, 8.4 \mathrm{~Hz} \mathrm{H}-6), 6.81(1 \mathrm{H}, d, J=8.4 \mathrm{~Hz}$, $\mathrm{H}-5), 6.224(1 \mathrm{H}, d, J=15.6 \mathrm{~Hz}, \mathrm{H}-8) .{ }^{13} \mathrm{C}-\mathrm{NMR}(100 \mathrm{MHz}$, Methanol- $d_{4}$ ) $\delta$ : 171.2 (C-9), 149.5 (C-4), 147.0 (C-7), 146.9 (C-3), 127.9 (C-1), 122.9 (C-6), 116.6 (C-5), 115.7 (C-8), $115.2(\mathrm{C}-2)$.

2.3.6. Rosmarinic Acid (6). White crystalline powder; HRESI-MS $m / z$ : 359.29[M-H]-. ${ }^{1} \mathrm{H}-\mathrm{NMR}(400 \mathrm{MHz}$, Methanol$\left.d_{4}\right) \delta: 7.51\left(1 \mathrm{H}, d, J=15.5 \mathrm{~Hz}, \mathrm{H}-7^{\prime}\right), 7.03(1 \mathrm{H}, d, J=2.0 \mathrm{~Hz}$, $\left.\mathrm{H}-2^{\prime}\right), 6.82\left(1 \mathrm{H}, \mathrm{dd}, J=2.0,8.5 \mathrm{~Hz}, \mathrm{H}-6^{\prime}\right), 6.72(1 \mathrm{H}, d$, $\left.J=8.5 \mathrm{~Hz}, \mathrm{H}-5^{\prime}\right), 6.66(1 \mathrm{H}, d, J=2.0 \mathrm{~Hz}, \mathrm{H}-2), 6.64(1 \mathrm{H}, d$, $J=8.0 \mathrm{~Hz}, \mathrm{H}-5), 6.57(1 \mathrm{H}, \mathrm{dd}, J=2.0,8 \mathrm{~Hz}, \mathrm{H}-6), 6.28(1 \mathrm{H}, d$, $\left.J=15.5 \mathrm{~Hz}, \mathrm{H}-8^{\prime}\right), 5.20(1 \mathrm{H}, \mathrm{dd}, J=5.0,7.5 \mathrm{~Hz}, \mathrm{H}-8), 3.06$ $(1 \mathrm{H}, \mathrm{dd}, J=5.5,14.5 \mathrm{~Hz}, \mathrm{H}-7 \mathrm{a}), 3.00(1 \mathrm{H}, \mathrm{dd}, J=5.5,14.5 \mathrm{~Hz}$, $\mathrm{H}-7 \mathrm{~b}) .{ }^{13} \mathrm{C}-\mathrm{NMR}\left(100 \mathrm{MHz}\right.$, Methanol- $\left.d_{4}\right) \delta: 171.3$ (C-9), $166.8\left(\mathrm{C}-9^{\prime}\right), 149.1\left(\mathrm{C}-4^{\prime}\right), 146 . .6\left(\mathrm{C}-7^{\prime}\right), 146.4\left(\mathrm{C}-3^{\prime}\right), 145.8$ (C-3), $144.9(\mathrm{C}-4), 129.1(\mathrm{C}-1), 127.3\left(\mathrm{C}-1^{\prime}\right), 121.5\left(\mathrm{C}-6^{\prime}\right)$, 117.3 (C-6), 116.6 (C-2), $115.6\left(\mathrm{C}-5^{\prime}\right), 116.4$ (C-5), 114.3 (C$\left.2^{\prime}\right), 114.2\left(\mathrm{C}-8^{\prime}\right), 73.8(\mathrm{C}-8), 37.0(\mathrm{C}-7)$.

2.3.7. Methyl Rosmarinate (7). White crystalline powder; HR-ESI-MS $m / z: \quad 373.24[\mathrm{M}-\mathrm{H}]-.{ }^{1} \mathrm{H}-\mathrm{NMR} \quad(400 \mathrm{MHz}$, Methanol- $\left.d_{4}\right) \delta: 7.55\left(1 \mathrm{H}, d, J=15.5 \mathrm{~Hz} \mathrm{H}-7^{\prime}\right), 7.04(1 \mathrm{H}, d$, $\left.J=2 \mathrm{~Hz}, \mathrm{H}-2^{\prime}\right), 6.95\left(1 \mathrm{H}, \mathrm{dd}, J=2.0,8.5 \mathrm{~Hz}, \mathrm{H}-6^{\prime}\right), 6.78(1 \mathrm{H}$, $\left.d, J=8.5 \mathrm{~Hz}, \mathrm{H}-5^{\prime}\right), 6.70(1 \mathrm{H}, d, J=2.0 \mathrm{~Hz}, \mathrm{H}-2), 6.69(1 \mathrm{H}, \mathrm{d}$, $J=8.0 \mathrm{~Hz}, \mathrm{H}-5), 6.57(1 \mathrm{H}, \mathrm{dd}, J=2.0,8.0 \mathrm{~Hz}, \mathrm{H}-6), 6.26(1 \mathrm{H}$, $\left.d, J=15.5 \mathrm{~Hz}, \mathrm{H}-8^{\prime}\right), 5.19(1 \mathrm{H}, \mathrm{dd}, J=5.0,7.5 \mathrm{~Hz}, \mathrm{H}-8), 3.7$ $\left(3 \mathrm{H}, \mathrm{s},-\mathrm{OCH}_{3}\right), 3.06(1 \mathrm{H}, \mathrm{dd}, J=5.5,14.5 \mathrm{~Hz}, \mathrm{H}-7 \mathrm{a}), 3.00$ $(1 \mathrm{H}, \mathrm{dd}, J=5.5,14.5 \mathrm{~Hz}, \mathrm{H}-7 \mathrm{~b}) .{ }^{13} \mathrm{C}-\mathrm{NMR} \quad(100 \mathrm{MHz}$, Methanol- $\left.d_{4}\right) \delta$ : $172.3(\mathrm{C}-9), 168.4\left(\mathrm{C}-9^{\prime}\right), 149.9\left(\mathrm{C}-4^{\prime}\right), 148.1$ $\left(\mathrm{C}-7^{\prime}\right), 146.9\left(\mathrm{C}-3^{\prime}\right), 146.3$ (C-3), $145.5(\mathrm{C}-4), 128.8$ (C-1), $127.7\left(\mathrm{C}-1^{\prime}\right), 123.3\left(\mathrm{C}-6^{\prime}\right), 121.9(\mathrm{C}-6), 117.6(\mathrm{C}-2), 116.6(\mathrm{C}-$ $\left.5^{\prime}\right), 116.4(\mathrm{C}-5), 115.3\left(\mathrm{C}-2^{\prime}\right), 114.2\left(\mathrm{C}-8^{\prime}\right), 74.8(\mathrm{C}-8), 52.8$ $\left(-\mathrm{OCH}_{3}\right), 38.0(\mathrm{C}-7)$.

2.3.8. Quercetin 3-O- $\beta$-D-Glucopyranoside (8). Yellow crystalline powder; HR-ESI-MS $m / z: 463.371[\mathrm{M}-\mathrm{H}]-.{ }^{1} \mathrm{H}-$ NMR $\left(400 \mathrm{MHz}\right.$, Methanol- $\left.d_{4}\right) \delta: 7.71(1 \mathrm{H}, d, J=1.6 \mathrm{~Hz}$, $\left.\mathrm{H}-2^{\prime}\right), 7.58\left(1 \mathrm{H}, \mathrm{dd}, J=1.2,8.4 \mathrm{~Hz}, \mathrm{H}-6^{\prime}\right), 6.87(1 \mathrm{H}, d$, $\left.J=8.4 \mathrm{~Hz}, \mathrm{H}-5^{\prime}\right), 6.39$ (1H, s, H-8), 6.20 (1H, s, H-6), 5.25 $(1 \mathrm{H}, \mathrm{d}, J=7.6 \mathrm{~Hz}$, galactose $\mathrm{H}-1), 3.37-3.60(4 \mathrm{H}, \mathrm{m}$, sugar protons). ${ }^{13} \mathrm{C}-\mathrm{NMR}\left(100 \mathrm{MHz}\right.$, Methanol- $\left.d_{4}\right) \delta: 179.6(\mathrm{C}-4)$, 166.1 (C-7), 163.2 (C-5), 159.1 (C-2), 158.5 (C-9), 149.9 (C$\left.4^{\prime}\right), 146.0\left(\mathrm{C}-3^{\prime}\right), 135.7(\mathrm{C}-3), 123.3\left(\mathrm{C}-6^{\prime}\right), 123.2\left(\mathrm{C}-1^{\prime}\right)$, $117.6\left(\mathrm{C}-5^{\prime}\right), 116.1\left(\mathrm{C}-2^{\prime}\right), 105.8(\mathrm{C}-10), 104.4$ (galactose-C1), 100.0 (C-6), 94.8 (C-8), 78.5 (galactose-C-3), 78.2 (galactose-C-5), 75.8 (galactose-C-2), 71.3 (galactose-C-4), 62.7 (galactose-C-6).

2.3.9. Luteolin (9). Yellow amorphous powder; HR-ESI-MS $m / z: 185.24[\mathrm{M}-\mathrm{H}]-.{ }^{1} \mathrm{H}-\mathrm{NMR}\left(400 \mathrm{MHz}, \mathrm{DMSO}-d_{6}\right) \delta: 12.97$ (s,-OH), $7.42\left(1 \mathrm{H}, d, J=1.6 \mathrm{~Hz}, \mathrm{H}-6^{\prime}\right), 7.39\left(1 \mathrm{H}, \mathrm{s}, \mathrm{H}-2^{\prime}\right), 6.88$ $\left(1 \mathrm{H}, d, J=8.4 \mathrm{~Hz}, \mathrm{H}-5^{\prime}\right), 6.66(1 \mathrm{H}, \mathrm{s}, \mathrm{H}-3), 6.43(1 \mathrm{H}, \mathrm{s}, \mathrm{H}-8)$, $6.18(1 \mathrm{H}, \mathrm{s}, \mathrm{H}-6) .{ }^{13} \mathrm{C}-\mathrm{NMR}\left(100 \mathrm{MHz}, \mathrm{DMSO}-d_{6}\right) \delta: 181.6(\mathrm{C}-$ 4), 164.1 (C-7), 163.8 (C-2), 161.4 (C-5), 157.2 (C-9), 149.7 (C$\left.4^{\prime}\right), 145.7\left(\mathrm{C}-3^{\prime}\right), 121.4\left(\mathrm{C}-1^{\prime}\right), 118.9\left(\mathrm{C}-6^{\prime}\right), 115.9\left(\mathrm{C}-5^{\prime}\right), 113.3$ (C-2' $), 103.6$ (C-10), 102.8 (C-3), 98.82 (C-6), 93.8 (C-8).

2.3.10. $3 \beta, 8 \beta, 12 \beta, 18$-Tetrahydroxy Pimar-15-en (10). White crystalline needles; freely soluble in methanol and ethyl acetate; HR-ESI-MS [M-H]- ion at $m / z 337.2354$ and molecular formula $\mathrm{C}_{20} \mathrm{H}_{34} \mathrm{O}_{4} \cdot{ }^{13} \mathrm{C}(100 \mathrm{MHz}, \mathrm{MeOD})$ and ${ }^{1} \mathrm{H}-\mathrm{NMR}$ (400 MHz, MeOD) spectroscopic data are given in Tables 1 and 2, respectively.

2.3.11. $7 \alpha, 8 \beta, 12 \beta, 18$-Tetrahydroxy Pimar-15-en (11). White crystalline needles, freely soluble in ethyl acetate and methanol; HR-ESI-MS [M-H] $]^{-}$ion at $\mathrm{m} / z 337.2322$ and molecular formula $\mathrm{C}_{20} \mathrm{H}_{34} \mathrm{O}_{4} \cdot{ }^{13} \mathrm{C}(100 \mathrm{MHz}, \mathrm{MeOD})$ and ${ }^{1} \mathrm{H}-\mathrm{NMR}$ (400 MHz, MeOD) spectroscopic data are given in Tables 1 and 2, respectively.

2.3.12. $3 \beta, 8 \beta, 11 \beta, 12 \alpha, 18$-Pentahydroxy Pimar-15-en (12). White crystalline needles; freely soluble in ethyl acetate and methanol, HR-ESI-MS [M-H]- ion at $\mathrm{m} / z 353.2269$ with molecular formula $\mathrm{C}_{20} \mathrm{H}_{34} \mathrm{O}_{5} \cdot{ }^{13} \mathrm{C}(100 \mathrm{MHz}, \mathrm{MeOD})$ and ${ }^{1} \mathrm{H}-\mathrm{NMR}(400 \mathrm{MHz}, \mathrm{MeOD})$ spectroscopic data are given in Tables 1 and 2, respectively.

2.3.13. $12 \beta$ Acetoxy, $8 \beta, 3 \beta, 18$-Trihydroxy Pimar-15-en (13). White crystalline needles; freely soluble in ethyl acetate and methanol, HR-ESI-MS $[\mathrm{M}-\mathrm{H}]^{-}$ion at $\mathrm{m} / z 379.2479$ with 
TABle 1: ${ }^{13} \mathrm{C}$-NMR spectroscopic data of compounds $10-14$ at $100 \mathrm{MHz}$.

\begin{tabular}{lccccc}
\hline $\mathrm{C}$ & $\delta_{\mathrm{C}}-10$ & $\delta_{\mathrm{C}}-11$ & $\delta_{\mathrm{C}}-12$ & $\delta_{\mathrm{C}^{-}} 13$ & $\delta_{\mathrm{C}^{-}}-14$ \\
\hline 1 & 36.7 & 37.7 & 37.7 & 36.6 & 35.1 \\
2 & 28.5 & 20.7 & 28.4 & 28.4 & 26.7 \\
3 & 68.5 & 43.5 & 68.4 & 68.1 & 65.9 \\
4 & 46.5 & 42.0 & 48.3 & 46.2 & 45.1 \\
5 & 43.9 & 44.4 & 43.4 & 45.2 & 43.3 \\
6 & 17.9 & 25.9 & 17.6 & 17.8 & 19.3 \\
7 & 39.2 & 75.1 & 39.0 & 39.0 & 39.3 \\
8 & 74.9 & 74.12 & 74.8 & 73.4 & 75.9 \\
9 & 40.5 & 48.0 & 44.2 & 41.6 & 39.8 \\
10 & 33.9 & 34.0 & 34.6 & 33.8 & 43.4 \\
11 & 27.1 & 27.4 & 73.1 & 24.2 & 26.3 \\
12 & 75.6 & 75.7 & 77.5 & 77.8 & 75.0 \\
13 & 37.7 & 41.5 & 38.34 & 37.6 & 37.33 \\
14 & 45.9 & 39.4 & 46.1 & 45.6 & 46.9 \\
15 & 153.4 & 149.7 & 153.4 & 153.0 & 153.4 \\
16 & 108.9 & 111.8 & 109.0 & 109.1 & 108.3 \\
$17-\mathrm{CH}_{3}$ & 33.7 & 33.9 & 33.4 & 33.6 & 34.1 \\
18 & 64.6 & 64.5 & 62.3 & 64.4 & 65.5 \\
$19-\mathrm{CH}_{3}$ & 25.0 & 26.1 & 25.1 & 25.0 & 24.6 \\
$20-\mathrm{CH}_{3}$ & 22.1 & 22.29 & 24.5 & 21.8 & 22.9 \\
$21-\mathrm{CO}^{-}$ & - & - & - & 172.2 & 171.1 \\
$22-\mathrm{OCH}_{3}$ & - & - & - & 21.4 & 21.1 \\
\hline
\end{tabular}

Note: compounds 10, 11, 12, and 13 were analyzed in methanol- $d_{4}$, Compound 14 was analyzed in acetone- $d_{6}$.

molecular formula $\mathrm{C}_{22} \mathrm{H}_{36} \mathrm{O}_{5} \cdot{ }^{13} \mathrm{C}(100 \mathrm{MHz}, \mathrm{MeOD})$ and ${ }^{1} \mathrm{H}-\mathrm{NMR}(400 \mathrm{MHz}, \mathrm{MeOD})$ spectroscopic data are given in Tables 1 and 2, respectively.

2.3.14. $3 \beta$ Acetoxy, $8 \beta, 12 \beta, 18$-Trihydroxy Pimar-15-en (14). White crystalline needles; freely soluble in acetone; sparingly soluble in ethyl acetate and methanol, HR-ESI-MS [M-H] ion at $m / z 379.2473$ with molecular formula $\mathrm{C}_{22} \mathrm{H}_{36} \mathrm{O}_{5} \cdot{ }^{13} \mathrm{C}$ $(100 \mathrm{MHz}, \mathrm{MeOD})$ and ${ }^{1} \mathrm{H}-\mathrm{NMR}\left(400 \mathrm{MHz}\right.$, Acetone- $\left.d_{6}\right)$ spectroscopic data are given in Tables 1 and 2, respectively.

\subsection{Antimicrobial Assay}

2.4.1. Microbial ATCC Strains. To investigate the in vitro antimicrobial potency of isolated new compounds, Grampositive bacteria S. aureus (ATCC 9144) and S. epidermidis (ATCC 12228) and Gram-negative bacteria ( $P$. aeruginosa (ATCC 27853) and E. coli (ATCC 14948) were purchased from National Path Lab, Butwal, Nepal. All the ATCC strains were subcultured on different culture media and investigated for Gram staining and biochemical test. The detail of microbial analysis of all the strains is given on supplementary data (Table S1).

2.4.2. Determination of Minimum Inhibitory Concentration (MIC). The twofold serial broth microdilution technique was adopted to calculate the MIC values of new isopimarane diterpenoids, against four different test organisms. A total of 10 vials were labeled and sterilized, then $960 \mu \mathrm{L}$ of sterilized Mueller-Hinton Broth (MHB) was transferred into each vial. For the sample solution preparation, $25000 \mu \mathrm{g} / \mathrm{mL}$ of stock solution was prepared in DMSO, subjected to serial dilution, using a 1:1 mixture of DMSO and water to prepare sample solutions of 10 different concentrations $(25000 \mu \mathrm{g} / \mathrm{mL}-48.8298125 \mathrm{mg} / \mathrm{mL})$. After that, $40 \mu \mathrm{L}$ of sample solution was transferred into a corresponding vial containing $960 \mu \mathrm{L}$ of $\mathrm{MHB}$, so that the final concentration of sample ranged from $1000 \mu \mathrm{g} / \mathrm{mL}$ to $1.95 \mu \mathrm{g} / \mathrm{mL}$. Bacteria with an inoculum of about $1 \times 10^{5} \mathrm{CFU} / \mathrm{mL}$ were loaded into each vial. For the preparation of microorganism inocula, broth culture was incubated for $12 \mathrm{~h}$, and turbidity of the suspension was adjusted to the turbidity of 0.5 McFarland standards. One inoculated vial was used as a negative control, to ensure suitability broth for growth of microorganism growth. Also, 4\% DMSO was tested as a blank control. Streptomycin sulfate and Vancomycin were considered as a positive control for Gram-negative and Grampositive microorganisms, respectively. After the incubation of the sample containing broth media for $24 \mathrm{~h}$ at $37^{\circ} \mathrm{C}$, the MIC value was determined. The MIC value was considered as the minimum concentration of compound that prevented the microorganism growth. The bacterial cell viability was determined by using 3(4,5 dimethylthiazol-2-yl)-2-5-diphenyl tetrazolium bromide (MTT) by incubating at $37^{\circ} \mathrm{C}$ for further $2 \mathrm{~h}$ and visual inspection of formazan formation [13].

\section{Results and Discussion}

3.1. Identification and Structure Elucidation of Noble Compounds. The air dried aerial parts of L. lucidus were macerated with methanol at normal room temperature. The methanol extract was subjected to successive fractionation between hexane, thyl acetate, n-butanol, and water. Reverse phase open column chromatographic analysis of the ethyl acetate fraction enabled the isolation of five new pimarane diterpenoids: $3 \beta, 8 \beta, 12 \beta$, 18-tetrahydroxy pimar-15-en (10), $7 \alpha, 8 \beta, 12 \beta, 18$-tetrahydroxy pimar-15-en (11), $3 \beta, 8 \beta, 11 \beta$, $12 \alpha$, 18-pentahydroxy pimar-15-en (12), $12 \beta$ acetoxy, $3 \beta, 8 \beta$, 18-trihydroxy pimar-15-en (13), and $3 \beta$ acetoxy, $8 \beta, 12 \beta, 18$ trihydroxy pimar-15-en (14), along with nine known compounds. Structure of known compounds was confirmed as protocatechuic acid (1) [14], protocatechualdehyde (2) [14], methyl 3,4 dihydroxy benzoate (3) [15], para-hydroxy benzoic acid (4) [16], caffeic acid (5) [17], rosmarinic acid (6) [4], methyl rosmarinate (7) [4], quercetin 3-O- $\beta$-D-glucopyranoside (8) [18], and luteolin (9) [4], through the comparison of obtained physical and spectroscopic data with previous literature. Chemical structures of all the isolated compounds are depicted in Figure 1.

Compound 10 appeared as white crystalline needles with HR-ESI-MS [M-H]- ion at $\mathrm{m} / z 337.2354$ and molecular formula $\mathrm{C}_{20} \mathrm{H}_{34} \mathrm{O}_{4}$. The ${ }^{1} \mathrm{H}$-NMR showed three olefin protons, twelve methylene groups, two oxygenated geminal methylene protons, two oxygenated methine protons, and two other normal methine protons, along with three methyl protons. The ${ }^{13} \mathrm{C}-\mathrm{NMR}$ exhibited 20 carbon signals consisting of two olefin carbon signals at $\delta_{\mathrm{C}} 153.4(\mathrm{C}-15)$ and 108.9 (C-16), four oxygenated carbon signals at $\delta_{\mathrm{C}} 75.6(\mathrm{C}-$ 12), 74.9 (C-8-tertiary carbon), 68.5 (C-3), and 64.5 (C-18). 
TABLE 2: ${ }^{1} \mathrm{H}-\mathrm{NMR}$ spectroscopic data of compounds $10-14$ at $400 \mathrm{MHz}$.

\begin{tabular}{|c|c|c|c|c|c|}
\hline $\mathrm{C}$ & ${ }^{1} \mathrm{H}-10$ & ${ }^{1} \mathrm{H}-11$ & ${ }^{1} \mathrm{H}-12$ & ${ }^{1} \mathrm{H}-13$ & ${ }^{1} \mathrm{H}-14$ \\
\hline \multirow[b]{2}{*}{1} & $1.70(1 \mathrm{H}, \mathrm{m}, \mathrm{H}-1 \mathrm{a})$ & $1.51(1 \mathrm{H}, \mathrm{m}, \mathrm{H}-1 \mathrm{a})$ & $1.83(1 \mathrm{H}, \mathrm{m}, \mathrm{H}-1 \mathrm{a})$ & $1.70(1 \mathrm{H}, \mathrm{m}, \mathrm{H}-1 \mathrm{a})$ & $1.81(1 \mathrm{H}, \mathrm{m}, \mathrm{H}-1 \mathrm{a})$ \\
\hline & $1.22(1 \mathrm{H}, \mathrm{m}, \mathrm{H}-1 \mathrm{~b})$ & $0.93(1 \mathrm{H}, \mathrm{m}, \mathrm{H}-1 \mathrm{~b})$ & $1.14(1 \mathrm{H}, \mathrm{m}, \mathrm{H}-1 \mathrm{~b})$ & $1.20(1 \mathrm{H}, \mathrm{m}, \mathrm{H}-1 \mathrm{~b})$ & $1.11(1 \mathrm{H}, \mathrm{m}, \mathrm{H}-1 \mathrm{~b})$ \\
\hline \multirow{2}{*}{2} & $1.94(1 \mathrm{H}, \mathrm{m}, \mathrm{H}-2 \mathrm{a})$ & $1.60(1 \mathrm{H}, \mathrm{m}, \mathrm{H}-2 \mathrm{a})$ & $1.97(1 \mathrm{H}, \mathrm{m}, \mathrm{H}-2 \mathrm{a})$ & $1.96(1 \mathrm{H}, \mathrm{m}, \mathrm{H}-2 \mathrm{a})$ & $1.83(1 \mathrm{H}, \mathrm{m}, \mathrm{H}-2 \mathrm{a})$ \\
\hline & $1.50(1 \mathrm{H}, \mathrm{m}, \mathrm{H}-2 \mathrm{~b})$ & $1.43(1 \mathrm{H}, \mathrm{m}, \mathrm{H}-2 \mathrm{~b})$ & $1.56(1 \mathrm{H}, \mathrm{m}, \mathrm{H}-2 \mathrm{~b})$ & $1.56(1 \mathrm{H}, \mathrm{m}, \mathrm{H}-2 \mathrm{~b})$ & $1.50(1 \mathrm{H}, \mathrm{m}, \mathrm{H}-2 \mathrm{~b})$ \\
\hline 3 & $3.39-3.42(1 \mathrm{H}, \mathrm{m}, \mathrm{H}-3)$ & $\begin{array}{c}1.46(1 \mathrm{H}, \mathrm{m}, \mathrm{H}-3 \mathrm{a} \\
1.27(1 \mathrm{H}, \mathrm{m}, \mathrm{H}-3 \mathrm{~b})\end{array}$ & $3.59(1 \mathrm{H}, \mathrm{t} \mathrm{H}-3)$ & $3.43(1 \mathrm{H}, \mathrm{m}, \mathrm{H}-3)$ & $4.1(1 \mathrm{H}, \mathrm{t}, \mathrm{H}-3)$ \\
\hline 5 & $\begin{array}{c}2.07(1 \mathrm{H}, \mathrm{dd}, 2.0,12.6 \\
\mathrm{Hz}, \mathrm{H}-5)\end{array}$ & $1.92(1 \mathrm{H}, \mathrm{m}, \mathrm{H}-5)$ & $\begin{array}{c}2.015(1 \mathrm{H}, \mathrm{dd}, 2.0 \\
12.6 \mathrm{~Hz}, \mathrm{H}-5)\end{array}$ & $\begin{array}{c}2.03(1 \mathrm{H}, \mathrm{dd}, 2.0 \\
12.6 \mathrm{~Hz}, \mathrm{H}-5)\end{array}$ & $1.96(1 \mathrm{H}, \mathrm{m}, \mathrm{H}-9)$ \\
\hline \multirow[b]{2}{*}{6} & $1.78(1 \mathrm{H}, \mathrm{m}, \mathrm{H}-6 \mathrm{a})$ & $2.04(1 \mathrm{H}, \mathrm{m}, \mathrm{H}-6 \mathrm{a})$ & $1.76(1 \mathrm{H}, \mathrm{m}, \mathrm{H}-6 \mathrm{a})$ & $1.76(1 \mathrm{H}, \mathrm{m}, \mathrm{H}-6 \mathrm{a})$ & $2.00(1 \mathrm{H}, \mathrm{m}-\mathrm{H} 6 \mathrm{a})$ \\
\hline & $1.45(1 \mathrm{H}, \mathrm{m}, \mathrm{H}-6 \mathrm{~b})$ & $1.54(1 \mathrm{H}, \mathrm{m}, \mathrm{H}-6 \mathrm{~b})$ & $1.48(1 \mathrm{H}, \mathrm{m}, \mathrm{H}-6 \mathrm{~b})$ & $1.45(1 \mathrm{H}, \mathrm{m}, \mathrm{H}-6$ & $1.70(1 \mathrm{H}, \mathrm{m}, \mathrm{H}-6 \mathrm{~b})$ \\
\hline 7 & $1.42(2 \mathrm{H}, \mathrm{m}, \mathrm{H}-7)$ & $3.57(1 \mathrm{H}, \mathrm{t}, \mathrm{H}-7)$ & $1.43(2 \mathrm{H}, \mathrm{m}, \mathrm{H}-7)$ & $1.50(2 \mathrm{H}, \mathrm{m}, \mathrm{H}-7)$ & $\begin{array}{l}1.42(1 \mathrm{H}, \mathrm{m}, \mathrm{H}-7 \mathrm{a}) \\
1.25(1 \mathrm{H}, \mathrm{m}, \mathrm{H}-7 \mathrm{~b})\end{array}$ \\
\hline 9 & $1.91(1 \mathrm{H}, \mathrm{m}, \mathrm{H}-9)$ & $1.65(1 \mathrm{H}, \mathrm{m}, \mathrm{H}-9)$ & $\begin{array}{c}2.042,(1 \mathrm{H}, \mathrm{d}, 2.8 \mathrm{~Hz} \\
\mathrm{H}-9)\end{array}$ & $1.84(1 \mathrm{H}, \mathrm{m}, \mathrm{H}-9)$ & $\begin{array}{c}2.15(1 \mathrm{H}, \mathrm{dd}, 2.0 \\
13.2 \mathrm{~Hz}, \mathrm{H}-5)\end{array}$ \\
\hline 11 & $\begin{array}{c}2.38(1 \mathrm{H}, \mathrm{td}, 2.8,2.4 \\
13.2 \mathrm{~Hz}, \mathrm{H}-11 \mathrm{a}) \\
1.56(1 \mathrm{H}, \mathrm{m}, \mathrm{H}-11 \mathrm{~b})\end{array}$ & $\begin{array}{c}2.41(1 \mathrm{H}, \mathrm{td}, 2.8,2.4 \\
13.2 \mathrm{~Hz}, \mathrm{H}-11 \mathrm{a}) \\
1.56(1 \mathrm{H}, \mathrm{m}-\mathrm{H} 11 \mathrm{~b})\end{array}$ & $4.1(1 \mathrm{H}, \mathrm{t}, \mathrm{H}-11)$ & $\begin{array}{c}2.39(1 \mathrm{H}, \mathrm{td}, 2.8,2.4 \\
13.2 \mathrm{~Hz}, \mathrm{H}-11 \mathrm{a} \\
1.66(1 \mathrm{H}, \mathrm{m}, \mathrm{H}-11 \mathrm{~b})\end{array}$ & $\begin{array}{l}1.93(1 \mathrm{H}, \mathrm{m}, \mathrm{H}-11 \mathrm{a}) \\
1.53(1 \mathrm{H}, \mathrm{m}, \mathrm{H}-11 \mathrm{~b})\end{array}$ \\
\hline 12 & $3.39-3.42(1 \mathrm{H}, \mathrm{m}, \mathrm{H}-12)$ & $3.48(1 \mathrm{H}, \mathrm{m}, \mathrm{H}-12)$ & $3.28(1 \mathrm{H}, \mathrm{m}, \mathrm{H}-12)$ & $4.62(1 \mathrm{H}, \mathrm{t}, \mathrm{H}-12)$ & $3.49(1 \mathrm{H}, \mathrm{m}, \mathrm{H}-12)$ \\
\hline 14 & $\begin{array}{c}2.015(1 \mathrm{H}, \mathrm{d}, 14.4 \mathrm{~Hz}, \mathrm{H}- \\
14 \mathrm{a}) \\
1.19(1 \mathrm{H}, \mathrm{m}, \mathrm{H}-14 \mathrm{~b})\end{array}$ & $\begin{array}{c}2.347(1 \mathrm{H}, \mathrm{d}, 14.4 \mathrm{~Hz}, \mathrm{H}- \\
14 \mathrm{a}) \\
1.098(1 \mathrm{H}, \mathrm{dd}, 0.8 \\
14.4 \mathrm{~Hz}, \mathrm{H}-14 \mathrm{~b})\end{array}$ & $\begin{array}{l}1.93(1 \mathrm{H}, \mathrm{m}, \mathrm{H}-14 \mathrm{a}) \\
1.26(1 \mathrm{H}, \mathrm{m}, \mathrm{H}-14 \mathrm{~b})\end{array}$ & $\begin{array}{l}1.63(1 \mathrm{H}, \mathrm{m}, \mathrm{H}-14 \mathrm{a}) \\
1.23(1 \mathrm{H}, \mathrm{m}, \mathrm{H}-14 \mathrm{~b})\end{array}$ & $\begin{array}{l}2.03(1 \mathrm{H}, \mathrm{m}, \mathrm{H}-14 \mathrm{a}) \\
1.15(1 \mathrm{H}, \mathrm{m}, \mathrm{H}-14 \mathrm{~b})\end{array}$ \\
\hline 15 & $\begin{array}{c}5.78(1 \mathrm{H}, \mathrm{dd}, 10.8, \\
17.2 \mathrm{~Hz}, \\
\mathrm{H}-15)\end{array}$ & $\begin{array}{l}5.91(1 \mathrm{H}, \mathrm{dd}, 10.8 \\
17.2 \mathrm{~Hz}, \mathrm{H}-15)\end{array}$ & $\begin{array}{c}5.76(1 \mathrm{H}, \mathrm{dd}, 10.8,17.2 \\
\text { HzH-15) }\end{array}$ & $\begin{array}{c}5.75(1 \mathrm{H}, \mathrm{dd}, 10.8 \\
17.2 \mathrm{~Hz}, \mathrm{H}-15)\end{array}$ & $\begin{array}{l}5.76(1 \mathrm{H}, \mathrm{dd}, 10.8 \\
17.2 \mathrm{~Hz}, \mathrm{H}-15)\end{array}$ \\
\hline 16 & $\begin{array}{c}4.90(1 \mathrm{H}, \mathrm{dd}, 1.2, \\
17.4 \mathrm{~Hz}, \mathrm{H}-16 \mathrm{a}) \\
4.79(1 \mathrm{H}, \mathrm{dd}, 1.2, \\
17.4 \mathrm{~Hz}, \mathrm{H}-16 \mathrm{~b})\end{array}$ & $\begin{array}{c}5.00(1 \mathrm{H}, \mathrm{dd}, 1.2, \\
14.2 \mathrm{~Hz}, \mathrm{H}-16 \mathrm{a}) \\
4.97(1 \mathrm{H}, \mathrm{dd}, 1.2,7.6 \mathrm{~Hz}, \\
\mathrm{H}-16 \mathrm{~b})\end{array}$ & $\begin{array}{c}4.88(1 \mathrm{H}, \mathrm{dd}, 1.2, \\
17.4 \mathrm{~Hz}, \mathrm{H}-16 \mathrm{a}) \\
4.79(1 \mathrm{H}, \mathrm{dd}, 1.2, \\
17.4 \mathrm{~Hz}, \mathrm{H}-16 \mathrm{~b})\end{array}$ & $\begin{array}{c}4.86(1 \mathrm{H}, \mathrm{dd}, 1.2,17.4 \mathrm{~Hz}, \\
\mathrm{H}-16 \mathrm{a}) \\
4.80(1 \mathrm{H}, \mathrm{dd}, 1.2 \\
17.4 \mathrm{~Hz}, \mathrm{H}-16 \mathrm{~b})\end{array}$ & $\begin{array}{c}4.84(1 \mathrm{H}, \mathrm{dd}, 1.2, \\
17.4 \mathrm{~Hz}, \\
\text { H-16a) } \\
4.74(1 \mathrm{H}, \mathrm{dd}, 1.2, \\
17.4 \mathrm{~Hz}, \mathrm{H}-16 \mathrm{~b})\end{array}$ \\
\hline $17-\mathrm{CH}_{3}$ & $0.92(3 \mathrm{H}, \mathrm{s}, \mathrm{H}-17)$ & $0.912(3 \mathrm{H}, \mathrm{s}, \mathrm{H}-17)$ & $1.01(3 \mathrm{H}, \mathrm{s}, \mathrm{H}-17)$ & $0.84(3 \mathrm{H}, \mathrm{s}, \mathrm{H}-17)$ & $0.88(3 \mathrm{H}, \mathrm{s}, \mathrm{H}-17)$ \\
\hline & $\begin{array}{c}3.89(1 \mathrm{H}, \mathrm{d}, 12.0 \mathrm{~Hz}, \mathrm{H}- \\
18 \mathrm{a})\end{array}$ & $\begin{array}{c}4.50(1 \mathrm{H}, \mathrm{d}, 12 \mathrm{~Hz}, \mathrm{H}- \\
18 \mathrm{a})\end{array}$ & $\begin{array}{c}3.85(1 \mathrm{H}, \mathrm{d}, 12.0 \mathrm{~Hz}, \mathrm{H}- \\
18 \mathrm{a})\end{array}$ & $\begin{array}{c}3.94(1 \mathrm{H}, \mathrm{d}, 12.0 \mathrm{~Hz}, \mathrm{H}- \\
18 \mathrm{a})\end{array}$ & $\begin{array}{c}5.08(1 \mathrm{H}, \mathrm{d}, 12.0 \mathrm{~Hz}, \\
\mathrm{H}-18 \mathrm{a})\end{array}$ \\
\hline 18 & $\begin{array}{c}3.54(1 \mathrm{H}, \mathrm{d}, 12.0 \mathrm{~Hz}, \mathrm{H}- \\
18 \mathrm{~b})\end{array}$ & $\begin{array}{c}3.487(1 \mathrm{H}, \mathrm{d}, 12 \mathrm{~Hz}, \mathrm{H}- \\
18 \mathrm{~b})\end{array}$ & $\begin{array}{c}3.54(1 \mathrm{H}, \mathrm{d}, 12.0 \mathrm{~Hz}, \mathrm{H}- \\
18 \mathrm{~b})\end{array}$ & $\begin{array}{c}3.56(1 \mathrm{H}, \mathrm{d}, 12.0 \mathrm{~Hz}, \mathrm{H}- \\
18 \mathrm{~b})\end{array}$ & $\begin{array}{c}4.52(1 \mathrm{H}, \mathrm{d}, 12.0 \mathrm{~Hz}, \\
\mathrm{H}-18 \mathrm{~b})\end{array}$ \\
\hline $19-\mathrm{CH}_{3}$ & $1.24(3 \mathrm{H}, \mathrm{s}, \mathrm{H}-19)$ & $1.26(3 \mathrm{H}, \mathrm{s}, \mathrm{H}-19)$ & $1.24(3 \mathrm{H}, \mathrm{s} \mathrm{H}-19)$ & $1.24(3 \mathrm{H}, \mathrm{s}, \mathrm{H}-19)$ & $1.23(3 \mathrm{H}, \mathrm{s}, \mathrm{H}-19)$ \\
\hline $20-\mathrm{CH}_{3}$ & $1.01(3 \mathrm{H}, \mathrm{s}, \mathrm{H}-20)$ & $1.01(3 \mathrm{H}, \mathrm{s}, \mathrm{H}-20)$ & $1.25(3 \mathrm{H}, \mathrm{s}, \mathrm{H}-20)$ & $1.00(3 \mathrm{H}, \mathrm{s}, \mathrm{H}-20)$ & $0.89(3 \mathrm{H}, \mathrm{s}, \mathrm{H}-20)$ \\
\hline $\begin{array}{l}22- \\
\mathrm{OCH}_{3}\end{array}$ & & & & $2.06(3 \mathrm{H}, \mathrm{s}, \mathrm{H}-22)$ & $1.98(3 \mathrm{H}, \mathrm{s}, \mathrm{H}-22)$ \\
\hline
\end{tabular}

Three methyl signals were detected at $\delta_{\mathrm{C}} 33.7$ (C-17), 25.0 (C19), and 22.1 (C-20). Similarly, six other normal methylene carbons at $\delta_{\mathrm{C}} 36.7(\mathrm{C}-1), 28.5(\mathrm{C}-2), 17.9$ (C-6), 39.2 (C-7), 27.1 (C-11), and 45.9 (C-14) and two normal methine carbon signals at $\delta_{\mathrm{C}} 43.9(\mathrm{C}-5)$ and 40.5 (C-9) were also recorded. Four carbon signals at $\delta_{\mathrm{C}} 74.8(\mathrm{C}-8), 45.5$ (C-4), 37.7 (C-13), and 33.9 (C-10) were found to be tertiary carbons through HMQC analysis. All protons were assigned to their corresponding carbons using HMQC experiment. Correlation of two oxygenated germinal methylene protons at $\delta_{\mathrm{H}} 3.89(1 \mathrm{H}$, $d, J=12.0 \mathrm{~Hz}, \mathrm{H}-18 \mathrm{a})$ and $3.54(1 \mathrm{H}, d, J=12.0 \mathrm{~Hz}, \mathrm{H}-18 \mathrm{~b})$ to carbon $\delta_{\mathrm{C}} 64.5(\mathrm{C}-18)$ suggests presence of $\mathrm{CH}_{2} \mathrm{OH}$ group. Among three olefinic protons, two were found to be connected with carbon $\delta_{\mathrm{C}} 153.4(\mathrm{C}-15)$. The presence of an ABX spin system in its ${ }^{1} \mathrm{H}$-NMR spectra due three vinyl protons of monosubstituted double bond at $d 4.9(\mathrm{dd}, J=1.2,17.4 \mathrm{~Hz}$, $\mathrm{H}-16 \mathrm{a}), d 4.79$ (dd, $J=1.2,17.4 \mathrm{~Hz}, \mathrm{H}-16 \mathrm{~b}$ ), and $d 5.78$ (dd, $J=10.8,17.2 \mathrm{~Hz}, \mathrm{H}-15)$ along with the respective carbon resonances in the ${ }^{13} \mathrm{C}-\mathrm{NMR}$ spectra $(\delta 153.4 \mathrm{ppm}, \mathrm{CH}$ and $d$
108.9 ppm $\mathrm{CH}_{2}$ ) ensured the compound is pimar-15-en derivative $[19,20]$. Analysis of the ${ }^{1} \mathrm{H}^{-1} \mathrm{H}$ COSY plot of compound (10) suggested correlations (H1/H2/H3), (H5/ H6/H7), (H9/H11/H12), and (H15/H16). In the HMBC data, correlations (C-11 and C-13)/H-12), (C-8/H-7), and ((C-18/ (H-5 and $\mathrm{H}-3)$ ) confirmed the positions of four hydroxyl groups; also carbon-proton correlations (C-17/H-15) and (C20/H-1) ensured the exact position of C-17 and C-20 methyl group, respectively. Presence of hydroxyl group at C-8 was confirmed as it was tertiary oxygenated carbon. Relative configuration of compound was confirmed by ROESY analysis. Configuration of $\mathrm{H}-17$ methyl group was found to be at $a$ position through the observation of difference in chemical shift of $a$ C-17 and $\beta$ C-17 in the literature [20-24]. Similarly, configuration of 8-hydroxyl group ensured to be at $\beta$ position by correlating with chemical shift of C-17 methyl group (trans configuration $\delta \mathrm{C}-17>32 \mathrm{ppm}$, cis relationships $\delta \mathrm{C}-17<25 \mathrm{ppm})[19-23]$. From ROESY, relative configuration of $\mathrm{H}-3, \mathrm{H}-5, \mathrm{H}-9, \mathrm{H}-12$, and $\mathrm{H}-20$ was confirmed 
<smiles>O=C(O)c1ccc(O)c(O)c1</smiles>

1<smiles>O=Cc1ccc(O)c(O)c1</smiles>

2<smiles>COC(=O)c1ccc(O)c(O)c1</smiles>

3<smiles>O=C(O)c1ccc(O)cc1</smiles>

4<smiles>O=C(O)/C=C/c1ccc(O)c(O)c1</smiles>

5<smiles>O=C(/C=C/c1ccc(O)c(O)c1)OC(Cc1ccc(O)c(O)c1)C(=O)O</smiles>

6<smiles>COC(=O)C(Cc1ccc(O)c(O)c1)OC(=O)/C=C/c1ccc(O)c(O)c1</smiles>

7<smiles>O=C1c2c(O)cc(O)cc2OC(c2ccc(O)c(O)c2)C1OC1OC(CO)C(O)C(O)C1O</smiles><smiles>C=C[C@]1(C)C[C@]2(O)CCC3C(C)(CO)[C@@H](O)CC[C@]3(C)[C@H]2[C@H](O)[C@@H](O)[C@@]1(C)C=C</smiles>

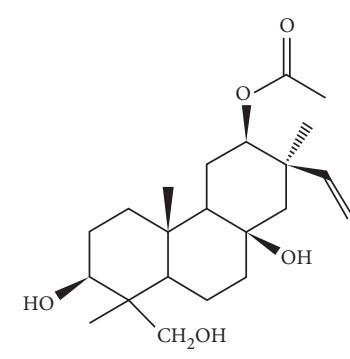

13<smiles>C=C[C@@]1(C)CCC2C(C)(C)[C@@H](OC(C)=O)CC[C@@]2(C)C[C@@H](O)[C@@H](O)C1</smiles>

14

FIgURE 1: Chemical structures of compounds isolated from Lycopus lucidus.

from the correlation as follows: (H-17 to $\mathrm{H}-11 \mathrm{~b}),(\mathrm{H}-11 \mathrm{~b}$ to $\mathrm{H}-12$ ), which confirmed that hydroxyl group at C-12 should be at $\beta$ position, correlation ( $\mathrm{H}-11 \mathrm{a}$ to $\mathrm{H}-20)$ ensured $\beta$ position of $\mathrm{H}-20$, correlations (H-11b to $\mathrm{H}-9$ ) and ( $\mathrm{H}-9$ to $\mathrm{H}-5)$ indicated $\alpha$ position of their proton, and finally correlations (H-9 to $\mathrm{H}-6 \mathrm{a}),(\mathrm{H}-6 \mathrm{a}$ to $\mathrm{H}-18 \mathrm{~b})$, and $(\mathrm{H}-18 \mathrm{~b}$ to $\mathrm{H}-3)$ ensured the $\beta$ position of $\mathrm{C}-3$ hydroxyl group. Therefore, chemical structure of compound $\mathbf{1 0}$ was established as $3 \beta, 8 \beta, 12 \beta, 18$-tetrahydroxy pimar-15-ene.

Compound 11 was obtained as white crystalline needles having HR-ESI-MS [M-H]- ion at $m / z 337.2322$ and molecular formula $\mathrm{C}_{20} \mathrm{H}_{34} \mathrm{O}_{4}$. It differs from compound 10 by presence of C-7-hydroxyl group instead of C-3. Hydroxylation at $\mathrm{C}-7$ was confirmed by its chemical shift $\left(\delta_{\mathrm{C}^{-}}-74.1\right)$. Final structure of compound is confirmed as $7 \alpha, 8 \beta, 12 \beta, 18$ tetrahydroxy pimar-15-ene.

Compound 12 was found to be white amorphous powder. Its molecular formula $\mathrm{C}_{20} \mathrm{H}_{34} \mathrm{O}_{5}$ was deduced by using HR-ESI-MS [M-H]- ion at $m / z$ 353.2269. Spectral data of compound 12 was similar to compound 10 except new addition of hydroxyl group at C-11, which was ensured by its chemical shift value $\left(\delta_{\mathrm{C}}-73.1\right)$. ROESY ensured the chemical structure of compound 12 as $3 \beta, 8 \beta, 11 \beta, 12 \alpha, 18$-pentahydroxy pimar-15-ene.

Compound 13 was white in color with crystalline needles. Its molecular formula $\mathrm{C}_{22} \mathrm{H}_{36} \mathrm{O}_{5}$ was deduced by using HR-ESI-MS [M-H]- ion at $\mathrm{m} / z$ 379.2479. Spectral data of compound 13 was different from compound 10 by addition of new methyl proton as an acetyl moiety at $\delta_{\mathrm{H}}$ $2.06(3 \mathrm{H}, \mathrm{s}, \mathrm{H}-22)$ along with one new tertiary carbonyl carbons signal at $\delta_{\mathrm{C}} 172.2(-\mathrm{CO}, \mathrm{C}-21)$ and one methyl carbon at $\delta_{\mathrm{H}} 22.4(\mathrm{C}-22)$. Increment of mass of compound 13 by 42 units $\left[-\mathrm{COCH}_{3}-\mathrm{H}^{+}\right]$also confirmed the addition of one acetyl moiety. Position of acetyl group was ensured accurately by other 2D-NMR analysis. In the HMBC data, carbon-proton correlations $(\mathrm{C}-21 / \mathrm{H}-22)$ illustrated attachment of the methyl group with carbonyl carbon. Correlation (C-12/H-21) verified the position of acetyl group. ROESY analysis illustrated the chemical name of compound 13 as $12 \beta$ acetoxy, $8 \beta, 3 \beta$, 18-trihydroxy pimar-15-ene. 
Compound $\mathbf{1 4}$ also was white crystalline needles having similar molecular formula, mass fragmentation, and spectral data with compound 13. It differed from compound $\mathbf{1 3}$ only by position of acetyl moiety. Chemical shift of C-3 $\left(\delta_{\mathrm{H}} 65.9\right)$ gives an idea about its oxygenation. From the HMBC data, carbon-proton correlations $(\mathrm{C}-21 / \mathrm{H}-22)$ illustrated that methyl group is attached with carbonyl carbon and another association (C-22/H-18) suggested the location of acetyl group at $\mathrm{C}-3$. Chemical name compound was deduced as $3 \beta$ acetoxy, $8 \beta, 12 \beta$, 18-trihydroxy pimar-15-ene. The ${ }^{13} \mathrm{C}$ and ${ }^{1} \mathrm{H}-\mathrm{NMR}$ spectroscopic data of compound $10-14$ are given in Tables 1 and 2, respectively.

\subsection{Antimicrobial Activity of Isolated Pimarane Diterpenoids.} The isolated new pimarane diterpenoids were examined for their antimicrobial potency in response to Grampositive bacteria $S$. epidermidis (ATCC 12228) and S. aureus (ATCC 9144) and Gram-negative bacteria $P$. aeruginosa (ATCC 27853) and E. coli (ATCC 14948), using a twofold serial broth dilution technique and MIC was evaluated. As shown in Table 3, the most significant compound was 10, which manifested MIC values of 15.62 and $31.25 \mu \mathrm{g} / \mathrm{mL}$ against $S$. epidermidis and $S$. aureus, respectively. Also, this compound was effective against $P$. aeruginosa and $E$. coli with MICs of $62.5 \mu \mathrm{g} / \mathrm{mL}$ and $125 \mu \mathrm{g} / \mathrm{mL}$, respectively. It is to be noted that compounds 13,14 , and 15 were ineffective against both Gram-negative strains at examined concentrations.

In the search for natural products as effective antimicrobial agents, numerous investigations have proved the promising bactericidal effect revealed by diterpenoids. However, very limited researches have been reported for the antibacterial potency of pimarane diterpenoids [25]. It has been reported that the presence of a decalin ring system in pimarane diterpenoids fascinates its penetration into the lipophilic cell membrane of bacteria to induce bacterial lysis. Furthermore, an appropriately positioned hydrophilic functional group (hydrogen bond donor group; $\mathrm{HBD}$ ) is capable of interacting with phosphorylated groups of the bacterial cell membrane [26]. In this study, MIC values of two isomers, i.e., compounds $\mathbf{1 0}$ and 11, were slightly different. The structural difference between these compounds is only the position of one hydroxyl group $\left(3^{\text {rd }}\right.$ position $\mathrm{OH}$ group in compound $\mathbf{1 0}$ is shifted to $7^{\text {th }}$ position in compound 11). Although the exact structureactivity relationship is not known, this may signify that a change in position of the same functional group may also alter the antibacterial effect. On the other hand, the antibacterial activity of compounds 13 and 14 was reported to be reduced, in which - $\mathrm{OH}$ group was replaced by an electron-withdrawing acetyl group. In a previous study, 3and 19-hydroxyl groups of isopimarane compounds were acetylated to investigate the role of substituents on antimicrobial activity. The results indicated that if acetylation occurs in the 3-hydroxyl position or both 19- and 3-hydroxyl groups, the antibacterial activity gets reduced significantly [27]. Hence, our study also revealed a similar result. Furthermore, this study suggested that a slight
TABLE 3: Results of the in vitro antibacterial activity (MIC) of compounds 10-14 against selected Gram-positive and Gramnegative bacteria.

\begin{tabular}{|c|c|c|c|c|c|c|c|}
\hline \multirow[t]{2}{*}{ Microorganisms } & \multicolumn{7}{|c|}{$\begin{array}{c}\text { Minimum inhibitory concentration } \\
(\mu \mathrm{g} / \mathrm{mL})\end{array}$} \\
\hline & 10 & 11 & 12 & 13 & 14 & Van & Strep \\
\hline $\begin{array}{l}\text { S. aureus } \\
\text { (ATCC 9144) }\end{array}$ & 31.25 & 31.25 & 62.5 & 125 & 125 & 0.488 & - \\
\hline $\begin{array}{l}\text { S. epidermidis } \\
\text { (ATCC } 12228 \text { ) }\end{array}$ & 15.62 & 31.25 & 125 & 250 & 250 & 0.488 & - \\
\hline $\begin{array}{l}P . \text { aeruginosa } \\
\text { (ATCC } 27853 \text { ) }\end{array}$ & 62.5 & 62.5 & 125 & - & - & - & 7.812 \\
\hline E. coli (ATCC 14948) & 125 & 250 & - & - & - & - & 7.812 \\
\hline
\end{tabular}

Van: Vancomycin hydrochloride; Strep: streptomycin sulfate; negative control: 4\% DMSO solution did not suppress the growth of the tested bacteria.

variation in the position and/or nature of the oxygenated moiety can result in a substantial change in antibacterial activity. Similar results were also found in previous studies, regarding the biological activities of other pimarane diterpenoids $[25,28,29]$.

\section{Conclusion}

Five new pimarane diterpenoids along with nine known compounds (polyphenol and flavonoids) were isolated from the ethyl acetate fraction of L. lucidus and their chemical structures were elucidated completely through instrumental data including 2D-NMR.

Among them, compound 6 (rosmarinic acid) was isolated in the largest amount. Compound 2 (protocatechualdehyde) and compound 4 (methyl 3, 4, dihydroxy benzoate) were isolated for the first time from this plant. Besides, antibacterial activity screening for newly isolated compounds showed that pimarane diterpenoids are more sensitive towards Gram-positive bacteria. Among the isolated new diterpenoids, compounds $\mathbf{1 0}$ and $\mathbf{1 1}$ were found to be most effective with $15.62 \mu \mathrm{g} / \mathrm{mL}$ and $31.25 \mu \mathrm{g} / \mathrm{mL}$ MICs values, respectively, against $S$. epidermidis. In addition, this screening showed that small changes in position and/or nature of the oxygenated functional group in diterpenoids can result in a significant variation in antibacterial activity.

\section{Data Availability}

All the data used to support the result of this research are available from Jitendra Pandey.

\section{Conflicts of Interest}

All the authors in this article have on conflicts of interest.

\section{Authors' Contributions}

Amrit Poudel and Hyeong Kyu Lee conceived the experiment. Jitendra Pandey designed and performed the experiment. Jitendra Pandey and Bang Yeon Hwang analyzed the 
data. Jitendra Pandey and Amrit Poudel wrote the manuscript. Jitendra Pandey revised the manuscript.

\section{Acknowledgments}

The authors are very much grateful to the Natural Medicine Research Centre, Korea Research Institute of Bioscience and Biotechnology, Cheongju-si 28116, and Chungbuk National University, Department of Pharmacy, Cheongju, Korea, for the technical support. The authors are also thankful to Mr. Ram Bahadur Khadka, Senior Microbiologist, Crimson College of Technology, Rupandehi, Nepal, for helping to provide microbial strains and their identification.

\section{Supplementary Materials}

The spectroscopic data used to support the findings of our research are available in Supplementary Figures S1-S35 and Table S1. (Supplementary Materials)

\section{References}

[1] H.-K. Moon and S.-P. Hong, "Nutlet morphology and anatomy of the genus Lycopus (Lamiaceae: mentheae)," Journal of Plant Research, vol. 119, no. 6, pp. 633-644, 2006.

[2] N. C. Henderson, "A taxonomic revision of the genus Lycopus (Labiatae)," The American Midland Naturalist, vol. 68, no. 1, pp. 95-138, 1962.

[3] H.-K. Moon, Y.-C. Kim, and S.-P. Hong, "Diagnostic characters and new populations of Lycopus lucidus var. hirtus (Lamiaceae)," Korean Journal of Plant Taxonomy, vol. 43, no. 2, pp. 99-102, 2013.

[4] E.-R. Woo and M. S. Piao, "Antioxidative constituents fromlycopus lucidus," Archives of Pharmacal Research, vol. 27, no. 2, pp. 173-176, 2004.

[5] T. Murata, M. Watahiki, Y. Tanaka, T. Miyase, and F. Yoshizaki, "Hyaluronidase inhibitors from takuran, Lycopus lucidus," Chemical and Pharmaceutical Bulletin, vol. 58, no. 3, pp. 394-397, 2010.

[6] S. Ślusarczyk, M. Hajnos, K. Skalicka-Woźniak, and A. Matkowski, "Antioxidant activity of polyphenols from Lycopus lucidus Turcz," Food Chemistry, vol. 113, pp. 134-138, 2009.

[7] X. Yang, Y. Zhao, N. He, and K. D. Croft, "Isolation, characterization, and immunological effects of $\alpha$-Galacto-oligosaccharides from a new source, the herb Lycopus lucidus turcz," Journal of Agricultural and Food Chemistry, vol. 58, no. 14, pp. 8253-8258, 2010.

[8] J.-Q. Yu, J.-C. Lei, X.-Q. Zhang et al., “Anticancer, antioxidant and antimicrobial activities of the essential oil of Lycopus lucidus Turcz. var. hirtus Regel," Food Chemistry, vol. 126, no. 4, pp. 1593-1598, 2011.

[9] X. Yang, Y. Lv, L. Tian, and Y. Zhao, "Composition and systemic immune activity of the polysaccharides from an herbal tea (Lycopus lucidusTurcz)," Journal of Agricultural and Food Chemistry, vol. 58, no. 10, pp. 6075-6080, 2010.

[10] J.-Y. Yang and H.-S. Lee, "Acaricidal activities of the active component of Lycopus lucidus oil and its derivatives against house dust and stored food mites (Arachnida: Acari)," Pest Management Science, vol. 68, no. 4, pp. 564-572, 2012.

[11] W. S. Lee, K.-R. Im, Y.-D. Park, N.-D. Sung, and T.-S. Jeong, "Human ACAT-1 and ACAT-2 inhibitory activities of pentacyclic triterpenes from the leaves of Lycopus lucidus
TURCZ," Biological and Pharmaceutical Bulletin, vol. 29, no. 2, pp. 382-384, 2006.

[12] S. Tiwari, S. Nepal, S. Sigdel et al., "Phytochemical screening, antibacterial-guided fractionation, and thin-layer chromatographic pattern of the extract obtained from Diploknema butyracea," Pharmacognosy Research, vol. 12, pp. 437-443, 2020.

[13] Í. D. Rebouças de Araújo, N. Coriolano de Aquino, A. C. Véras de Aguiar Guerra et al., "Chemical composition and evaluation of the antibacterial and Cytotoxic activities of the essential oil from the leaves of Myracrodruon urundeuva," BMC Complementary and Alternative Medicine, vol. 17, pp. 1-8, 2017.

[14] B. A. Ayinde, D. N. Onwukaeme, and E. K. Omogbai, "Isolation and characterization of two phenolic compounds from the stem bark of Musanga cecropioides R. Brown (Moraceae)," Acta Poloniae Pharmaceutica, vol. 64, pp. 183-185, 2007.

[15] T. Tsuda, M. Watanabe, K. Ohshima, A. Yamamoto, S. Kawakishi, and T. Osawa, "Antioxidative components isolated from the seed of tamarind (Tamarindus indica L.)," Journal of Agricultural and Food Chemistry, vol. 42, no. 12, pp. 2671-2674, 1994.

[16] Azizuddin, T. Makhmoor, and M. I. Choudhary, "Radical scavenging potential of compounds isolated from Vitex agnus-castus," Turk journal of chemistry, vol. 34, pp. 119-126, 2010.

[17] H.-Y. Park, M.-H. Nam, H.-S. Lee, W. Jun, S. Hendrich, and K.-W. Lee, "Isolation of caffeic acid from Perilla frutescens and its role in enhancing $\gamma$-glutamylcysteine synthetase activity and glutathione level," Food Chemistry, vol. 119, no. 2, pp. 724-730, 2010.

[18] Y. Shokoohinia, M. Rashidi, L. Hosseinzadeh, and Z. Jelodarian, "Quercetin-3-O- $\beta$-d-glucopyranoside, a dietary flavonoid, protects PC12 cells from $\mathrm{H} 2 \mathrm{O} 2$-induced cytotoxicity through inhibition of reactive oxygen species," Food Chemistry, vol. 167, pp. 162-167, 2015.

[19] T. Meragelman, G. L. Silva, E. Mongelli, and R. R. Gil, "Entpimarane type diterpenes from Gnaphalium gaudichaudianum," Phytochemistry, vol. 62, no. 4, pp. 569-572, 2003.

[20] A. M. L. Seca, D. C. G. A. Pinto, and A. M. S. Silva, "Structural elucidation of pimarane and isopimarane diterpenoids: the 13C NMR contribution," Natural product communications, vol. 3, Article ID 1934578X0800300317, 2008.

[21] M. G. d. Carvalho, J. S. Alves, E. V. L. da-Cunha, J. M. Barbosa-Filho, and M. S. D. Silva, "Pimarane diterpenes and a sesquiterpene from Salzmmania nitida," Anais da Academia Brasileira de Ciências, vol. 78, no. 1, pp. 17-21, 2006.

[22] Y. Takaishi, K. Miyagi, K. Kawazoe, K. Nakano, K. Li, and H. Duan, "Terpenoids from Tripterygium wilfordii var. regelii," Phytochemistry, vol. 45, no. 5, pp. 975-978, 1997.

[23] X.-D. Wu, L.-C. Zhang, J. He et al., "Two new diterpenoids fromExcoecaria acerifolia," Journal of Asian Natural Products Research, vol. 15, no. 2, pp. 151-157, 2013.

[24] F. Bohlmann and C. Zdero, "Sandaracopimarene derivatives from Senecio subrubriflorus," Phytochemistry, vol. 21, no. 7, pp. 1697-1700, 1982.

[25] P. Rijo, M. F. Simões, A. Duarte, and B. Rodríguez, "Isopimarane diterpenoids from Aeollanthus rydingianus and their antimicrobial activity," Phytochemistry, vol. 70, no. 9, pp. 1161-1165, 2009.

[26] T. S. Porto, N. A. J. C. Furtado, V. C. G. Heleno et al., "Antimicrobial ent-pimarane diterpenes from Viguiera 
arenaria against gram-positive bacteria," Fitoterapia, vol. 80, no. 7, pp. 432-436, 2009.

[27] V. M. S. Isca, J. Andrade, A. S. Fernandes et al., "In vitro antimicrobial activity of isopimarane-type diterpenoids," Molecules, vol. 25, no. 18, p. 4250, 2020.

[28] J. R. Hanson, "Diterpenoids," Natural Product Reports, vol. 21, no. 2, pp. 312-320, 2004.

[29] J. R. Hanson and R. Diterpenoids, "Diterpenoids," Natural Product Reports, vol. 23, no. 6, pp. 875-885, 2006. 